\title{
Controlled high-fidelity navigation in the charge stability diagram of a double quantum dot
}

\author{
Diego S. Acosta Coden* and Rodolfo H. Romero \\ Instituto de Modelado e Innovación Tecnológica (CONICET-UNNE) \\ and Facultad de Ciencias Exactas y Naturales y Agrimensura, \\ Avenida Libertad 5400, W3404AAS Corrientes, Argentina \\ Esa Räsänen \\ Department of Physics, Tampere University of Technology, P.O. Box 692, FI-33101 Tampere, Finland
}

(Dated: November 5, 2014)

\begin{abstract}
We propose an efficient control protocol for charge transfer in a double quantum dot. We consider numerically a two-dimensional model system, where the quantum dots are subjected to timedependent electric fields corresponding to experimental gate voltages. Our protocol enables navigation in the charge stability diagram from a state to another through controllable variation of the fields. We show that the well-known adiabatic Landau-Zener transition - when supplemented with a time-dependent field tailored with optimal control theory - can remarkably improve the transition speed. The results also lead to a simple control scheme that requires only a single parameter obtained from the experimental charge stability diagram. Eventually, we can control the system at the fastest speed allowed by the quantum dynamics and with a high fidelity.
\end{abstract}

\section{INTRODUCTION}

High-fidelity control of electron dynamics in a quantum system is of paramount importance for quantum information processing [1]. The quantum information, encoded in qubits, is processed by the action of quantum gates that must act accurately on time scales much shorter than the coherence time and under the fault-tolerance threshold [2]. In addition, the experimental limitations and the quantum speed limit (QSL) [3, 4] (a fundamental limit imposed by quantum mechanics) determine, respectively, the possible temporal dependencies and minimum time duration of realistic quantum gates. In the quantum control terminology, the action of a quantum gate corresponds to engineering the time dependence of external parameters to drive the qubit from an initial state to a desired target state. Such constrained quantum control problems can be successfully stated into the general theoretical framework of quantum optimal control theory (OCT) [5, 6].

Semiconductor qubits, embodied by charge or spin degrees of freedom of electrons in quantum dots, are in the heart of the current qubit proposals due to their potential scalability within present solid-state technology. Particularly, the most used setup for semiconductor qubits consists of two coupled semiconductor quantum dots where the confinement potential and charge state can be controlled with high accuracy [7]. In principle, charge or spin qubits can be manipulated by both optical and electrical methods. However, all-electrical control by local gates seems more appealing than optical control due to practical difficulties in focusing optimally shaped laser beams with the desired spatial precision. For example, in the

\footnotetext{
* diegoacoden@gmail.com
}

double quantum dots (DQD) the charge degree of freedom is fully tunable by biasing the double well potential and interdot tunnel barrier by gate voltages [9].

Since the seminal Letter of Grossmann et al. [8] a number of theoretical and experimental works have addressed the issue of controlling the localization of an electron state in a double well potential [9-22]. These various control strategies include the use of analytically wellestablished phenomena like the paradigmatic LandauZener (LZ) transition [12-14], Landau-Zener-Stückelberg interferometry $[15,16]$, the composite pulse (CP) protocol and several other two-level control strategies [12, 2427], and OCT [18-23]. For the experimental control strategies, as can be seen in [10, 15-17], the first stage is the measurement of the charge stability diagram (CSD). This diagram characterizes the electron localization as functions of the gate voltages [7] and therefore initializes the scenario for the control strategy. However, unlike experimental control strategies, this diagram has rarely been applied in the context of theoretical control schemes.

In this work, we use OCT to numerically find electric fields (and thus the corresponding gate voltages) that lead to desired charge transfer in a semiconductor double quantum dot. We compare the efficiency of the OCT method with the LZ scheme, one of the simplest but slowest strategies, and also with the CP protocol that allows driving the system at the QSL [24, 27]. We find that the optimal gate voltages tailored to produce the charge transfer between the dots are fully parameterizable through a one-parameter trajectory in the charge stability diagram (CSD) defined by the degeneracy point of the regions of none and one electron occupation, and by the initial and final configurations. Thus, the relevant information to produce charge transfer between the dots can be extracted directly from the CSD. This remarkable result is in contrast with the most of the alterna- 
tive strategies in which further information of the energy spectrum is required.

The rest of the paper is organized as follows. In Sec. II we first describe the physical model and parameters and then explain with the dynamics in the adiabatic and diabatic basis. This is followed by the description of the OCT scheme and field parameters. In Sec. III we focus on our results by first comparing the fidelity and speed of the three protocols. Then we propose parametric scheme defined in the CSD. Finally, in Sec. IV we discuss the advantages of the proposed mechanism.

\section{THEORY AND METHODS}

\section{A. Model}

The effective-mass Hamiltonian for the $N$-electron system is given by

$$
H=\sum_{i=1}^{N} h\left(\mathbf{r}_{i}\right)+\sum_{i<j}^{N} C\left(\mathbf{r}_{i}, \mathbf{r}_{j}\right),
$$

where the single-particle Hamiltonian

$$
h(\mathbf{r})=-\frac{1}{2 m^{*}} \nabla^{2}+V(\mathbf{r})+V_{b}(\mathbf{r})+W(\mathbf{r}),
$$

consists of the kinetic-energy term, DQD model potential $V(\mathbf{r})$, central potential barrier $V_{b}(\mathbf{r})$ and a surrounding boundary $W(\mathbf{r})$ (see below). The second term in the Hamiltonian,

$$
C\left(\mathbf{r}_{i}, \mathbf{r}_{j}\right)=\frac{e^{2}}{4 \pi \varepsilon \varepsilon_{0}\left|\mathbf{r}_{i}-\mathbf{r}_{j}\right|},
$$

describes the Coulombic interaction between the electrons. The complete Hamiltonian is used to build the CSD, but we focus on time-dependent transitions occurring within the one-electron regions. We use the material parameters of GaAs, i.e., the effective mass $m^{*}=0.067$ $m_{e}$ and the dielectric constant $\varepsilon=12.4$.

Inspired by the realistic experimental setup of coupled quantum dots [10], we propose a model potential composed by a double well

$$
V(x, y, t)=-\frac{V_{L}(t)}{e^{\left(r_{L}-a\right) / b}+1}-\frac{V_{R}(t)}{e^{\left(r_{R}-a\right) / b}+1},
$$

where the depths of the left and right wells, $V_{L}(t)$ and $V_{R}(t)$, are time-dependent functions corresponding to the applied gate voltages. Here $a$ is the radius of the dot, $b$ defines the hardness of the boundary, $r_{L}=$ $\sqrt{(x \mp d)^{2}+y^{2}}$ is the distance from the center of right or left dot, and $d$ is a half of the distance between the centers of the dots. The parameter values are set to $a=40 \mathrm{~nm}$, $b=1 \mathrm{~nm}$, and $d=60 \mathrm{~nm}$. This potential is supplemented by a central potential barrier $V_{b}(\mathbf{r})=v_{b} /\left(1+e^{\left(x-b_{x}\right) / b}\right)$ to separate the QDs as well as by a rectangular boundary defined as

$$
W(\mathbf{r})=2-\frac{1}{1+e^{\left(|x|-l_{x}\right) / b}}-\frac{1}{1+e^{\left(|y|-l_{y}\right) / b}} .
$$

This potential prevents the wave function to extend over the external leads. We use values $l_{x}=180 \mathrm{~nm}$ and $l_{y}=$ $100 \mathrm{~nm}$ corresponding to an area of $360 \mathrm{~nm} \times 200 \mathrm{~nm}$. A potential barrier height between the dots is $v_{b}=0.565$ meV.

The spatial eigenstates of the single-particle problem are expanded in a basis set of Gaussian functions centered at the dot positions

$$
G_{i}(\mathbf{r})=\mathcal{N} x^{m_{i}} y^{n_{i}} \exp \left(-\alpha_{i} r_{Q_{i}}^{2}\right) \quad \text { with } Q_{i}=\mathrm{R}, \mathrm{L},
$$

where $\mathcal{N}$ is a normalization constant, $\alpha_{i}$ are the Gaussian exponents, and $m_{i}$ and $n_{i}$ are integers that define the $z$ projection $\ell_{i}=m_{i}+n_{i}$ of the angular momentum of the basis function [35]. For the two-particle case we consider only the singlet states that is always the ground state in the absence of an external magnetic field [36]. Then the spatial eigenstates are expanded in properly symmetrized products of Gaussian functions

$\Phi_{i j}\left(\mathbf{r}_{1}, \mathbf{r}_{2}\right)=\left\{\begin{array}{cc}\frac{1}{\sqrt{2}}\left[G_{i}\left(\mathbf{r}_{1}\right) G_{j}\left(\mathbf{r}_{2}\right)+G_{j}\left(\mathbf{r}_{1}\right) G_{i}\left(\mathbf{r}_{2}\right)\right], & \text { for } i \neq j \\ G_{i}\left(\mathbf{r}_{1}\right) G_{i}\left(\mathbf{r}_{2}\right), & \text { for } i=j\end{array}\right.$

of the form

$$
\Psi_{m}\left(\mathbf{r}_{1}, \mathbf{r}_{2}\right)=\sum_{n=1}^{N_{\text {conf }}} c_{m n} \Phi_{n}\left(\mathbf{r}_{1}, \mathbf{r}_{2}\right)
$$

where $N_{\text {conf }}$ is the number of two-electron configurations and $c_{m n}$ are the expansion coefficients. The eigenenergies and the corresponding eigenfunctions of the oneand two-particle problem are obtained by using the Ritzvariational method for a non-orthogonal basis set. For each pair $\left(V_{L}, V_{R}\right)$ the exponents $\alpha_{i}$ are optimized simultaneously using Nelder-Mead method [37] to minimize the ground-state energy. To achieve converged energy results for the ground state a set of $2 s 2 p 2 d$ basis is found to be sufficient in all the cases considered here and all two-electron configurations for such a basis is included.

\section{B. Dynamics in the adiabatic and diabatic basis}

Our goal is to control the state vector of the system $|\Psi(t)\rangle$ by varying the gate voltages $V_{L}(t)$ and $V_{R}(t)$ with time. For a given pair of functions $\left[V_{L}(t), V_{R}(t)\right]$ the evolution of the state vector is governed by the timedependent Schrödinger equation (TDSE)

$$
i \frac{\partial|\Psi(t)\rangle}{\partial t}=\hat{H}(t)|\Psi(t)\rangle
$$

where $|\Psi(t)\rangle$ is the state vector of the system and $\hat{H}(t)$ is a simplified notation for the variation in time through the gate voltages of the Hamiltonian, see Eq. (1). 
The state vector $|\Psi(t)\rangle$ can be expanded in any set of eigenvectors of the Hilbert space, in particular, in the so-called diabatic basis, which is the stationary basis of the system, involving the eigenvectors of the timeindependent part of the Hamiltonian $\hat{H}_{0}$,

$$
\hat{H}_{0}\left|\Phi_{i}^{D}\right\rangle=\varepsilon_{i}^{D}\left|\Phi_{i}^{D}\right\rangle,
$$

where $\hat{H}(t)=\hat{H}_{0}+\hat{U}(t)$. The expansion of the state vector in the diabatic basis is

$$
|\Psi(t)\rangle=\sum_{i} D_{i}(t) e^{-\imath \varepsilon_{i}^{D} t}\left|\Phi_{i}^{D}\right\rangle,
$$

and the dynamics is governed by the time evolution of the expansion coefficients $D_{i}(t)$,

$$
\dot{D}_{i}(t)=-\imath \sum_{j} D_{j}(t) e^{-\imath\left(\varepsilon_{j}^{D}-\varepsilon_{i}^{D}\right) t} U_{i j}(t),
$$

where $U_{i j}(t)=\left\langle\Phi_{i}^{D}|\hat{U}(t)| \Phi_{j}^{D}\right\rangle$.

Another possible basis set, that facilitates the discussion of time-evolving quantum systems, is the adiabatic basis made of the eigenstates of the instantaneous Hamiltonian,

$$
\hat{H}(t)\left|\Phi_{i}^{A}(t)\right\rangle=\varepsilon_{i}^{A}(t)\left|\Phi_{i}^{A}(t)\right\rangle,
$$

where the eigenvalues $\varepsilon_{i}^{A}(t)$ and the eigenstates $\left|\Phi_{i}^{A}(t)\right\rangle$ are the instantaneous eigenvalues and eigenvectors of the Hamiltonian at time $t$, i.e., for a given $V_{L}(t)$ and $V_{R}(t)$. The expansion of the state vector in the adiabatic basis,

$$
|\Psi(t)\rangle=\sum_{i} A_{i}(t)\left|\Phi^{A}(t)\right\rangle,
$$

leads to the following equation for the expansion coefficients $A_{i}(t)$

$$
\dot{A}_{j}(t)=-\imath \sum_{i k k^{\prime}}\left[R_{j k}^{-1} H_{k k^{\prime}} R_{k^{\prime} i}-\imath R_{j k}^{-1} \dot{R}_{k i}\right] A_{i}(t),
$$

where $H_{i j}=\left\langle\Phi_{i}^{A}(t)|\hat{H}(t)| \Phi_{j}^{A}(t)\right\rangle, R_{i j}$ connects the superposition coefficients of the state vector expansions (11) and (14)

$$
D_{i}(t)=\sum_{j} R_{i j}(t) A_{j}(t)
$$

and $R_{i j}^{-1}(t)$ is the matrix of the diabatic-adiabatic transformation

$$
\left|\Phi_{i}^{A}(t)\right\rangle=\sum_{j} R_{i j}^{-1}(t)\left|\Phi_{j}^{D}\right\rangle .
$$

The systems of differential equations for the coefficients $D_{i}(t)$ and $A_{i}(t)$, given by equations (12) and (15), respectively, is integrated numerically with the fourth-order Runge-Kutta method [38]. The matrices $R^{-1}$ and $R$ depend on time through the gate voltages $V_{L}(t)$ and $V_{R}(t)$, and are thus available at an arbitrary time instant for the integrator. We calculate these matrices on a grid in a given region of the CSD and we use a simple first-order polynomial interpolation for every point between the grid points. A spacing of $0.01 \mathrm{meV}$ between grid points has been shows to be sufficiently small to obtain converged dynamics on the adiabatic basis. In terms of the number of levels, we find converged dynamics with the first eight diabatic eigenstates for the diabatic evolution, and with the first two adiabatic eigenstates for the adiabatic evolution.

\section{Optimal control theory}

If we assume that the system is initially, at $t=0$, in the state $\left|\Phi_{I}\right\rangle$, the key task of OCT is to determine the functions $V_{L}(t)$ and $V_{R}(t)$ in order to get, after a time $T$, a predefined final state $\left|\Phi_{F}\right\rangle$. For this purpose, a cost functional $J$, that will be subsequently maximized, is constructed:

$$
J\left[\Psi, \chi, V_{L}, V_{R}\right]=J_{1}[\Psi]+J_{2}\left[V_{L}\right]+J_{2}\left[V_{R}\right]+J_{3}\left[\Psi, \chi, V_{L}, V_{R}\right] .
$$

Here the part $J_{1}[\Psi]=\left|\left\langle\Psi(T) \mid \Phi_{F}\right\rangle\right|^{2}$ maximizes the projection of the wave function after the pulse duration $T$ on the target state $\left|\Phi_{F}\right\rangle$. This quantity is usually designated as the yield. The second functional

$$
J_{2}[V]=-\alpha\left[\frac{1}{T} \int_{0}^{T} d t V^{2}(t) / s(t)-f\right],
$$

restricts the total fluence $f$ of the gate voltage $V(t)$. Here $\alpha$ is a time-independent Lagrange multiplier, and $s(t)$ serves as an envelope function for the field. In this paper we use an envelope function of a form [21]

$$
s(t)=\frac{1}{2}\left\{\operatorname{erf}\left[\frac{a}{T}\left(t-\frac{T}{b}\right)\right]+\operatorname{erf}\left[\frac{-a}{T}\left(t-T+\frac{T}{b}\right)\right]\right\}
$$

with $a=5.8$ ps and $b=2.9$ ps. The constraint that the electronic wave function has to satisfy the TDSE, formulated in integral form, is expressed in terms of the Lagrange multiplier $\chi(t)$ by the third functional

$$
J_{3}\left[\Psi, \chi, V_{L}, V_{R}\right]=-2 \operatorname{Im} \int_{0}^{T} d t\left\langle\chi(t)\left|\left(i \partial_{t}-\hat{H}(t)\right)\right| \Psi(t)\right\rangle .
$$

In what follows we split the field functions into two terms, $V_{L}=v_{L}^{i}+v_{L}$ and $V_{R}=v_{R}^{i}+v_{R}$, where $v^{i}$ is a fixed initial ansatz taking into account the available information on the physical system (structure of avoided crossings, previous control protocols, etc.), and $v$ is optimized with the OCT algorithm. In particular, we define $v_{L}^{i}(t)$ and $v_{R}^{i}(t)$ as linear functions on time in accordance to the $\mathrm{LZ}$ recipe detailed in the following. In this scheme, Eqs. (18), (19), and (21) can be restated in terms of $v_{L}$ and $v_{R}$ instead of $V_{L}$ and $V_{R}$. 
Setting the variations in the total functional to zero, i.e., $\delta J=0$, leads to the so-called control equations

$$
\begin{aligned}
i \partial_{t}|\Psi(t)\rangle & =\hat{H}\left(v_{L}, v_{R}, t\right)|\Psi(t)\rangle, \quad|\Psi(0)\rangle=\left|\Phi_{I}\right\rangle, \\
i \partial_{t}|\chi(t)\rangle & =\hat{H}\left(v_{L}, v_{R}, t\right)|\chi(t)\rangle, \quad|\chi(T)\rangle=\left|\Phi_{F}\right\rangle, \\
v_{L}(t) & =-\frac{1}{\alpha_{L}} \operatorname{Im}\left\langle\chi(t)\left|\frac{-1}{e^{\left(r_{L}-a\right) / b}+1}\right| \Psi(t)\right\rangle, \\
v_{R}(t) & =-\frac{1}{\alpha_{R}} \operatorname{Im}\left\langle\chi(t)\left|\frac{-1}{e^{\left(r_{R}-a\right) / b}+1}\right| \Psi(t)\right\rangle .
\end{aligned}
$$

In order to solve the above nonlinear coupled equations we use an iterative algorithm proposed by Werschnik and Gross [30]. The algorithm allows us to introduce further experimental constraints, in particular, the filter frequency $\omega_{c}$ defined as the maximum allowed frequency in the optimized pulse.

\section{RESULTS}

\section{A. Charge stability diagram}

The CSD [Fig. 1(a)] specifies the charge configuration of the DQD as a function of potential gates in terms of the pair $\left(n_{L}, n_{R}\right)$ where $n_{L}\left(n_{R}\right)$ represents the number of electrons in the left (right) dot. The regions with electron localization in the left or right dot are separated by a degeneracy line, where the electrons occupy orbitals that span both dots. The charge configuration in each region is determined from the condition that the electrochemical potential in the dots $\mu\left(n_{L}+n_{R}\right)$, defined as

$$
\mu\left(n_{L}+n_{R}\right)=E_{G}\left(n_{L}+n_{R}\right)-E_{G}\left(n_{L}+n_{R}-1\right),
$$

where $E_{G}(n)$ represent the ground state energy of the DQD with $n$ electrons, is equal to the electrochemical potential in the reservoirs which are defined as zero.

The system setup with the total potential $V$ and its components are visualized in Fig. 1(b) (at $y=0$; note that the real potential is two-dimensional). The CSD as a function of the gate voltages $V_{L}$ and $V_{R}$ are shown in Fig. 1(a), where the degeneracy lines (black solid lines) are associated with the passage of an electron between the reservoir and the left and right dot (horizontal and vertical lines), or with the exchange of one electron between the dots (diagonal line). On the diagonal there are two points (gray), where the one-electron configurations $(1,0)$ and $(0,1)$ are equally probable with $(0,0)$ at $\sim 0.53$ $\mathrm{meV}$ or with $(1,1)$ at $\sim 2 \mathrm{meV}$. In the following these points are referred to as shallow and deep degeneracy points, respectively.

The black dots marked on the CSD in Fig. 1(a) correspond to two configurations that will be used as our initial or final states of the control protocol. The first point, located at $\left(V_{L}, V_{R}\right)=(1.8,0.9) \mathrm{meV}$, has a Hamiltoninan and ground state denoted by the ket $H_{L}$ and $|L\rangle$ respectively, while for the second point, located at $\left(V_{L}, V_{R}\right)=(0.9,1.8) \mathrm{meV}$, the Hamiltonian and ground state are denoted by $H_{R}$ and $|R\rangle$. The red dashed line corresponds to one of the possible paths on the CSD to connect the states $|L\rangle$ and $|R\rangle$. This trajectory corresponds to a linear relation of the voltages $V_{L}$ and $V_{R}$ associated with the LZ transition between $|L\rangle$ and $|R\rangle$. In Fig. 1(c) we show the eigenenergies of the first two eigenstates over this LZ path as a function of the detuning $\varepsilon=V_{R}-V_{L}$. As expected, the energies are clearly separated at large detunings. The positive (negative) slope of the eigenstate is related to its left (right) dominant localization. At $\varepsilon=0$ the energies form a clear avoided crossing, which has a central role in the LZ charge transfer process.

Our CSD in Fig. 1(a) is in accordance with previous CSDs calculated from a microscopic theory [32, 33]. In contrast to these studies, in which only the doublewell potential is considered, we require that the DQD eigenstates are confined in a region of the size of the lithographics dimensions of the sample through $W(r)$ in Eq. (5). This spatial restriction affects mainly the weakly bound states and the main effect of this potential in the CSD is modify the position of the degeneracy points. For example, the shallow degeneracy point calculated in the absence of the potential well $W(r)$ is moved at $0.43 \mathrm{meV}$, i.e., $0.1 \mathrm{meV}$ below its previous value.

\section{B. Efficiency and speed of control protocols for CSD navigation}

We consider three control strategies to vary $V_{L}(t)$ and $V_{R}(t)$, each corresponding to a different pathway through the CSD. We may consider the charge localization in the left or right dot, together with a transition between the states $|L\rangle$ and $|R\rangle$ as a simple single-qubit operation. For realistic material parameters and device dimensions used in this work, the operating time should be kept within the maximum coherence time of GaAs of about $7 \mathrm{~ns}$ [40] and the frequency spectrum of the functions $V_{L}(t)$ and $V_{R}(t)$ should not exceed the typical high-frequency limit of operation with gate electrodes, around $50 \mathrm{GHz}$ [39].

First, we consider the passage of the electron using a LZ transition [12]. Now, the depth of the left dot decreases linearly in time while the depth of the right dot increases at the same rate, i.e., $V_{L, R}^{\mathrm{LZ}}(t)=V_{L, R}^{0} \pm\left(V_{R}^{0}-\right.$ $\left.V_{L}^{0}\right) t / T$, where $V_{L}^{0}=1.8 \mathrm{meV}$ and $V_{R}^{0}=0.9 \mathrm{meV}$.

The second scheme we consider is the CP protocol [25]. This scheme has been proved to be capable to control a system described by a LZ-type Hamiltonian at the maximum speed allowed by quantum mechanics $[24,26]$. In addition, the CP scheme corresponds to an analytical solution of the optimal control problem for these systems [26]. In the CP protocol, the time dependence of the gate 

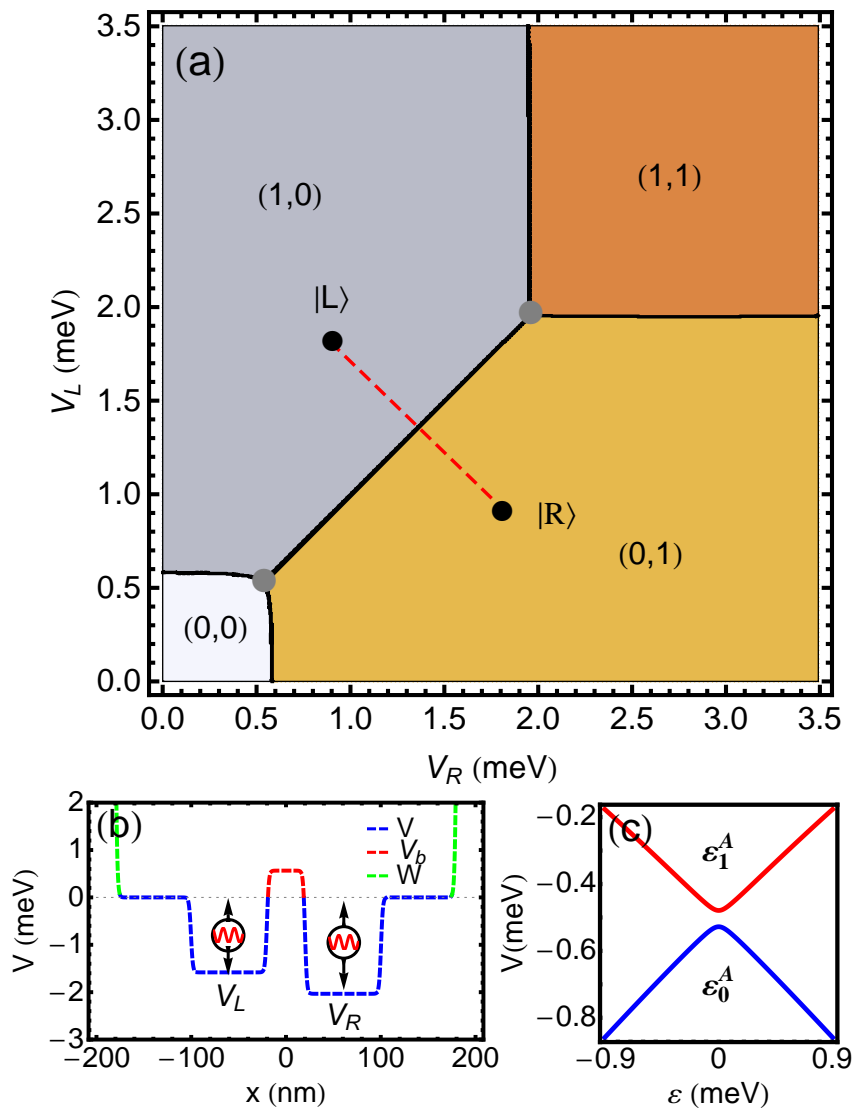

FIG. 1. (a) Charge stability diagram for our double quantum dot system. The notation $\left(n_{1}, n_{2}\right)$ identifies the dominant localization of the ground state and characterizes the regions of the charge stability diagram. These regions are separated by degeneracy lines (black solid lines) and the intersection of two of these lines is called degeneracy point (gray points). The black points, located at $(0.9,1.8) \mathrm{meV}$ and $(1.8,0.9) \mathrm{meV}$ and denoted by the kets $|L\rangle$ and $|R\rangle$ respectively, correspond to the initial and final state of the control protocol. The red dashed line represents one possible pathway on the CSD, related to the LZ strategy, to go from $|L\rangle$ to $|R\rangle$. (b) Total potential $V(\mathbf{r})+V_{b}(\mathbf{r})+W(\mathbf{r})$ as a function of $x$ (for $y=0$ ) colored according to its leading contribution. (c) The energies of the ground and excited states as a function of the detuning $\varepsilon\left(\varepsilon=V_{R}-V_{L}\right)$ varied from the configuration $(1,0)$ to $(0,1)$ through the dashed red line from $|L\rangle$ to $|R\rangle$.

voltages is a piecewise function given by

$$
\varepsilon(t)=\left\{\begin{array}{cc}
-\varepsilon_{0} & t \leq 0 \\
+\varepsilon_{M} & t \in\left(0, t_{0}\right] \\
0 & t \in\left(t_{0}, T-t_{0}\right) \\
-\varepsilon_{M} & t \in\left[T-t_{0}, T\right) \\
+\varepsilon_{0} & t \geq 0
\end{array}\right.
$$

where $\varepsilon_{0}=0.9 \mathrm{meV}, \varepsilon_{M}=20 \mathrm{meV}$, and $t_{0}$ satisfies $\varepsilon_{M} t_{0}=\pi / 4$. In the first and last piece, the gate voltages are chosen such that the initial $|L\rangle$ and final $|R\rangle$ states are eigenstates of the instantaneous Hamiltonian for $t=0$ and $t=T$, respectively. In between these intervals, there are two $\delta$-like pulses separated by a central piece with
$V_{L}=V_{R}$. It should be noted that the abrupt changes in the field make it rather unrealistic for experimental applications.

Finally, we propose a third scheme in which the linear LZ gate voltages $V_{L, R}^{\mathrm{LZ}}(t)$ are corrected by optimized functions $v_{L, R}(t)$ obtained with OCT. The composite voltages have a form

$$
V_{i}(t)=V_{i}^{\mathrm{LZ}}(t)+v_{i}(t), \quad(i=L, R) .
$$

While the LZ protocol is known to produce slow (adiabatic) transitions, the optimized part in Eq. (27) enables extremely fast, non-adiabatic, transitions as shown below.
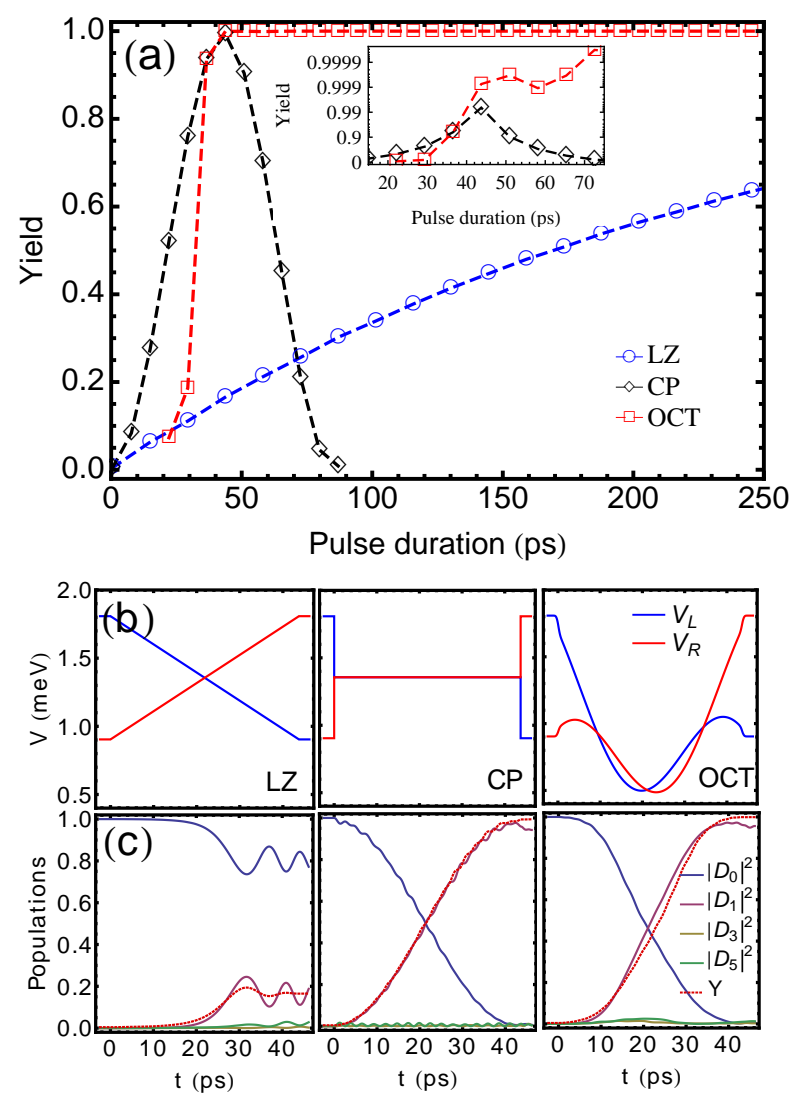

FIG. 2. (a) Calculated yield as a function of the pulse duration for the Landau-Zener (LZ), composite pulse (CP) and Optimized Landau-Zener (OCT) protocols. The inset shows a more detailed comparison between $\mathrm{CP}$ and OCT, both reaching yields higher than $99 \%$ for pulse lengths of 44 ps. The OCT yield keeps on increasing up to $99.99 \%$ at about 74 ps. (b) Time variation of left and right voltages as a function of time for LZ, CP and OCT, when the pulse length is fixed to 44 ps. (c) Diabatic electron populations in left and right dots as a function of time for LZ, CP and OCT procedures.

Figure 2(a) shows the yield as a function of the field length for the transition $|L\rangle \rightarrow|R\rangle$ obtained with LZ, CP, and OCT strategies. The TDSE have been solved in the diabatic basis of eigenstates of the left localized Hamiltoninan $H_{L}$ and the OCT calculations are carried out 
with a total fluence of $0.31 \mathrm{mev}^{2}$. The CP and OCT protocols allow efficient transitions leading to yields higher than $99 \%$ for the field length of $44 \mathrm{ps}$, while the LZ protocol requires about 1000 ps to reach a comparable fidelity. It should be noted [see the inset of Fig. 2(a)] that the OCT field can reach even higher yields up to $\sim 99.99 \%$ with longer fields. The remarkable fact that the CP and the OCT protocols give the same minimal pulse duration to control the charge with a high fidelity is related to the property of these strategies to drive the system at the QSL $[27,41]$. The coincidence between these very different strategies suggest that the time of $44 \mathrm{ps}$ is intrinsic of our system. For that pulse duration the gate voltages and the populations in the relevant diabatic states as a function of time are shown in Figure 2(b-c). At that characteristic time the LZ process leads to only minor population transfer, whereas the $\mathrm{CP}$ and OCT methods show a similar trend of fast transition from the initial to the target configuration.

Short transition times (below 100 ps) obtained here with the CP and OCT schemes are naturally favorable for applications of DQDs in the coherent regime. One of the key questions is the ability to produce the suggested fields with experimental methods. In this respect, the OCT field in Fig. 2(b) looks rather and smooth, which has also been found in earlier studies on electrically controlled DQD transitions [42]. On the other hand, the abrupt changes characteristic of the CP field are difficult to obtain with experimental methods.

Next we examine in detail different optimized paths in the CSD. Fig. 3(a) shows curves in the plane $\left(V_{L}(t), V_{R}(t)\right)$ obtained by an OCT optimization of the left and right well depths [Eq. (27)]. Several fixed values for the fluence between 0 and $0.31 \mathrm{meV}^{2}$ are considered, and the time is set to 44 ps. Notably, the OCT algorithm for the maximization of the yield provokes $V_{L}(t)$ and $V_{R}(t)$ to evolve along a loop-shaped trajectory, which is axially symmetric with respect to the diagonal $V_{L}=V_{R}$. The trajectories give rise to fidelities increasing with the fluence, from $16.5 \%$ (LZ process, straight line) up to $99.9 \%$. It should be noted that the increasing fidelity is related with an approach of the trajectory towards the shallow degeneracy point. In particular, the highest fidelity corresponds to the time-dependent gate voltages for which the trajectory passes through the triple point of degeneracy [34].

The instantaneous energies, $E_{0}^{A}$ and $E_{1}^{A}$, for the ground and excited states calculated with this field are shown in Fig. 3(b). It shows that the effect of the timedependent gate voltages is to get these levels closer to each other during the interval 8 ps $\lesssim t \lesssim 36$ ps, thus favoring the interference between the states. This regime corresponds to trajectories close to the degeneracy line separating the $(1,0)$ and $(0,1)$ regions. This effect is also reflected in the populations of the ground and excited levels as a function of time shown in Fig. 3(c). In the above mentioned time interval, both levels are, on the average, equally populated reaching the final occupations
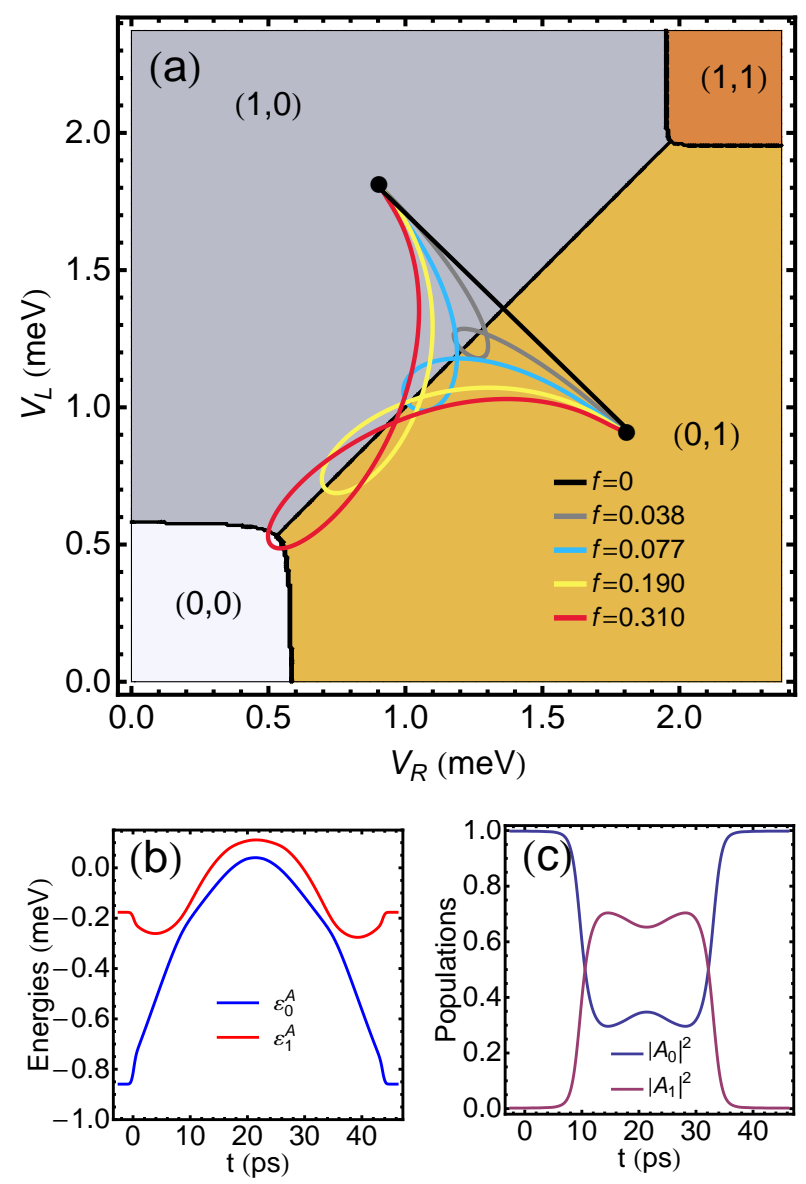

FIG. 3. (a) Optimized trajectories in the charge stability diagram for various values of fluence in $\mathrm{meV}^{2}$ for a pulse duration of $44 \mathrm{ps}$. For the larger fluence, the trajectory passes through the degeneracy point at the corner of the region $(0,0)$. For the red trajectory the energies of the adiabatic ground and exited states as a function of the time and their populations are shown in (b) and (c), respectively.

in the last 8 ps of the field.

As a conclusion of this section, it should be pointed out that the OCT strategy, characterized by loop-shaped trajectories in the CSD, is shown to be able to produce charge transfer at nearly the QSL, en tanto es medido por el CP protocol, thus fulfilling the stringent requirements for the quantum error correction (loss of fidelity ranging between $0.01 \%$ and fractions of a percent $[28,29]$ ).

\section{Control protocol from a parametric ansatz}

Based on the above OCT results, we may propose a control protocol depending on a single parameter, $A_{\varepsilon}$, whose meaning is explained below. Similarly to Eq. (27) above, the detuning $\varepsilon(t)=V_{R}(t)-V_{L}(t)$ and the sum $\mu(t)=V_{R}(t)+V_{L}(t)$ can be expressed as

$$
\varepsilon(t)=\varepsilon^{\mathrm{LZ}}(t)+\varepsilon^{\mathrm{OCT}}(t)
$$




$$
\mu(t)=\mu^{\mathrm{LZ}}(t)+\mu^{\mathrm{OCT}}(t) .
$$

Since the optimized field is set to zero at $t=0$ and $t=T$ by definition, the same condition applies also to $\mu(t)$. We suggest the following ansatz,

$$
\begin{aligned}
\varepsilon(t) & =\varepsilon_{L Z}(t)+A_{\varepsilon} \sin (2 \pi t / T) \\
\mu(t) & =\mu_{0}+A_{\mu}[\cos (2 \pi t / T)-1],
\end{aligned}
$$

where $A_{\varepsilon}$ and $A_{\mu}$ are fitting parameters. As discussed in the previous section, the trajectories passing close to the degeneracy point (DP), $\left(V_{L}, V_{R}\right)=\left(V_{D P}, V_{D P}\right)$, have yields higher than $99 \%$. Therefore, we fix the value of $A_{\mu}$ by the condition that the degeneracy point is the turning point at $t=T / 2$; therefore, $A_{\mu}=\left(\mu_{0}-2 V_{D P}\right) / 2$ with $\mu_{0}=V_{L}(0)+V_{R}(0)$. This ansatz, with such a parametrization, gives a very accurate representation of the OCT protocol for a variety of trajectories.

In Fig. 4(a) we show three different parametrized transitions (A, B, C) marked in the CSD [for the parameter values see Figs. 4(b-d)]. The protocol is applied to reach the target state symmetrically localized in the right dot from the initial state localized in the left dot. In all cases, the trajectories are smooth curves that drive the system to the desired target state with a high fidelity.

In Figs. 4(b-d) we show the yield as a function of the pulse duration for the three parametrized trajectories $\mathrm{A}, \mathrm{B}$ and $\mathrm{C}$ and for the CP strategy. Note that, for a given trajectory and value of $A_{\epsilon}$, our control strategy has several characteristic times when the maximum yield is obtained. The shortest of these characteristic times, which is obtained by adjusting the value of $A_{\epsilon}$, is greater than the time in which the maximum yield is obtained by the CP. As with the case of the optimized pulses, the parametrized pulses can at best match the speed of the CP for the optimal value of the parameters $A_{\epsilon}$. For the processes $\mathrm{A}, \mathrm{B}$ and $\mathrm{C}$ we found that this optimal parameters correspond to $A_{\epsilon}=0.452,0.791$ and $1.085 \mathrm{meV}$, respectively. In Figs. 4(b-d) we also show the yields for pulses with different values of $A_{\epsilon}$. For the A trajectory a fidelity of $98 \%$ is obtained at the characteristic time measured by the CP. For this trajectory our scheme leads to a second maximum, at around four times the characteristic time, to a fidelity of 99.9\%. For trajectories $\mathrm{B}$ and $\mathrm{C}$ the proposed protocol produces yields as high as $99.99 \%$ within times similar to the $\mathrm{CP}$ one with the optimal values of $A_{\epsilon}$.

\section{CONCLUDING REMARKS}

We have studied the optimal procedures to control charge transitions in a realistic double quantum dot by using driving gate voltages. We have shown that a field tailored with quantum optimal control theory on top of the Landau-Zener protocol is an efficient strategy to speed up the transitions without compromising the fidelity. Moreover, the optimal fields are found to be rel-
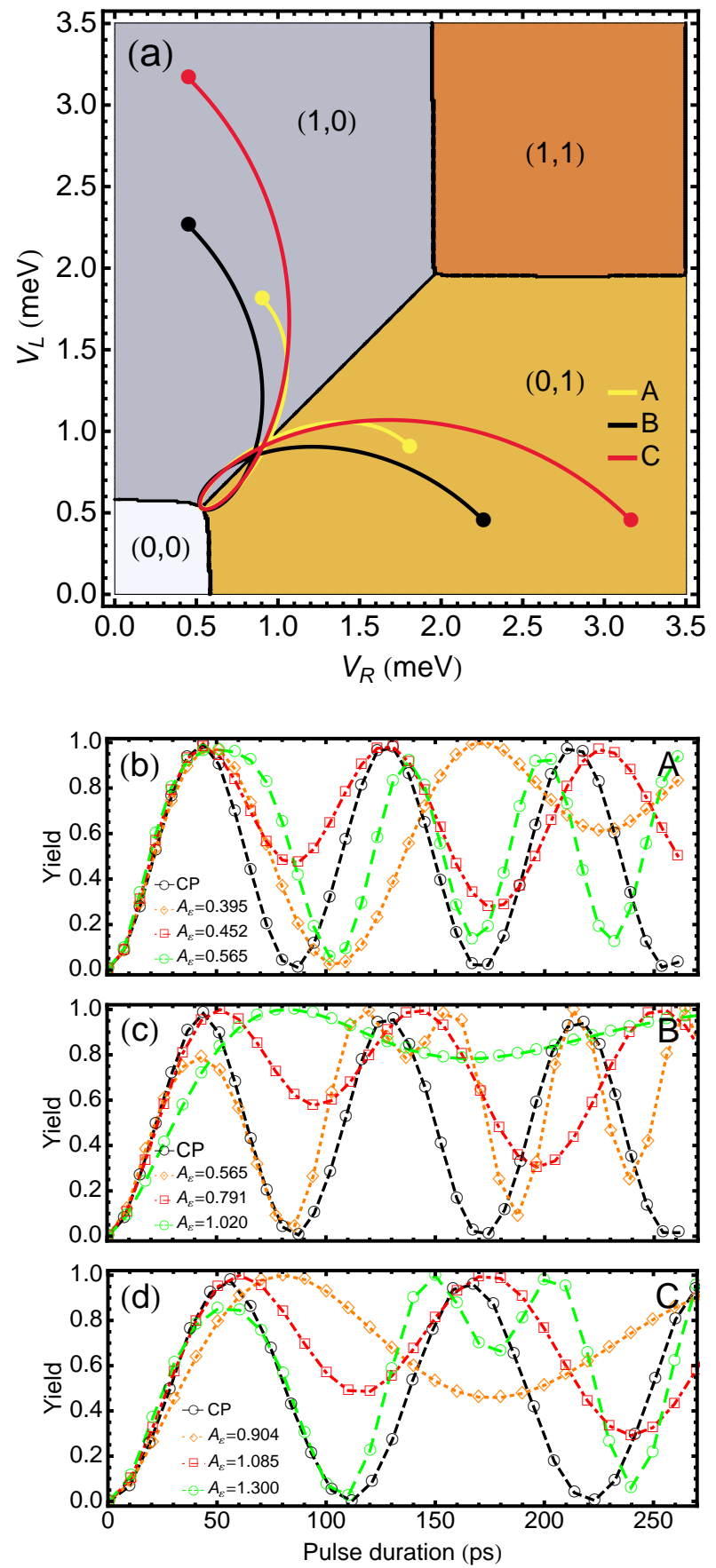

FIG. 4. (a) Trajectories for the proposed parametrized protocol in Eqs. (30) and (31) for the optimal value of $A_{\epsilon}$ in meV calculated fixing the pulse duration at the time in wich the $\mathrm{CP}$ reaches its first maximum value. The initial states for the $\mathrm{A}, \mathrm{B}$ and $\mathrm{C}$ trajectories are the ground states for the gate voltages $\left(V_{L}, V_{R}\right)=(1.8,0.9) \mathrm{meV},(2.26,0.45) \mathrm{meV}$ and $(3.16,0.45) \mathrm{meV}$ respectively. (b-d) Yield as a function of the pulse duration for the trajectories in the charge stability diagram for points $\mathrm{A}, \mathrm{B}$ and $\mathrm{C}$ with three different values of $A_{\epsilon}$. The result of the composite pulse (CP) protocol is shown for comparison. 
atively smoothly varying and thus realistic in comparison with step-like fields proposed by the composite pulse method. The transition times in the optimized and composite pulse protocols correspond to the quantum speed limit.

The analysis of the dynamics in the charge stability diagram shows that the evolution follows loop-shaped trajectories. They correspond to time-dependent gate voltages for which the charge states of the system stays, most of the time, close to the degeneracy line, where the electron is partially localized in both quantum dots. Such a condition has been found to allow an efficient transition with a high yield. Finally, we have been able to find simple single-parameter fits for the optimal fields, which in principle could be implemented in an experimental setup.

\section{ACKNOWLEDGMENTS}

We acknowledge CONICET, ANPCyT (Argentina) and SGCyT-UNNE (Argentina) for partial financial support of this project through grants PICT 2012-2866 and F007-2011. The project was also supported by the Academy of Finland, COST Action CM1204 (XLIC), Nordic Innovation through its Top-Level Research Initiative (project no. P-13053), and the European Community's FP7 through the CRONOS project, grant agreement no. 280879.
[1] M. A. Nielsen and I. L. Chuang, Quantum computation and quantum information, Cambridge University Press, Cambridge (2000).

[2] D. A. Lidar,T. A. Brun, Quantum Error Correction, Cambridge University Press, Cambridge (2013).

[3] P. Pfeifer, Phys. Rev. Lett. 70, 3365 (1993).

[4] L. B. Levitin and T. Toffoli, arXiv:0905.3417.

[5] A.P. Peirce, M.A. Dahleh, and H. Rabitz, Phys. Rev. A 37, 4950 (1988); I.R. Sola, J. Santamaria, and D.J. Tannor, J. Phys. Chem. A 102, 4301 (1998).

[6] V. F. Krotov, Global Methods in Optimal Control Theory (Marcel Dekker, New York, 1996).

[7] R. Hanson, L. P. Kouwenhoven, J. R. Petta, S. Tarucha, and L. M. K. Vandersypen, Rev. Mod. Phys. 79, 1217 (2007).

[8] F. Grossmann, T. Dittrich, P. Jung, and P. Hanggi, Phys. Rev. Lett. 67, 516 (1991).

[9] T. Hayashi, T. Fujisawa, H. D. Cheong, Y. H. Jeong, and Y. Hirayama, Phys. Rev. Lett. 91, 226804 (2003).

[10] J. R. Petta, A. C. Johnson, C. M. Marcus, M. P. Hanson, and A. C. Gossard, Phys. Rev. Lett. 93, 186802 (2004).

[11] J. Gorman, D. G. Hasko, and D. A. Williams, Phys. Rev. Lett. 95, 090502 (2005).

[12] C. Zener, Proc. R. Soc. A 137, 696 (1932).

[13] G. E. Murgida, D. A. Wisniacki, and P. I. Tamborenea, Phys. Rev. Lett. 99, 036806 (2007).

[14] G. E. Murgida, D. A. Wisniacki, and P. I. Tamborenea, Phys. Rev. B 79, 035326 (2009).

[15] G. Cao, H.-O. Li, T. Tu, L. Wang, Ch. Zhou, M. Xiao, G.-C. Guo, H.-W. Jiang and G.P. Guo, Nat. Commun. 4, 1401 (2013).

[16] J. Stehlik, Y. Dovzhenko, J. R. Petta, J. R. Johansson, F. Nori, H. Lu, and A. C. Gossard, Phys. Rev. B 86, 121303(R) (2012).

[17] Y. Dovzhenko, J. Stehlik, K. D. Petersson, J. R. Petta, H. Lu, and A. C. Gossard, Phys. Rev. B (RC) 84, 161302 (2011).

[18] S. G. Kosionis, A. F. Terzis, and E. Paspalakis, Phys. Rev. B 75, 193305 (2007).

[19] E. Räsänen, A. Castro, J. Werschnik, A. Rubio, and E. K. U. Gross, Phys. Rev. B 77, 085324 (2008).

[20] R. Nepstad, L. Sælen, I. Degani, and J. P. Hansen, J. Phys.: Condens. Matter 21, 215501 (2009).
[21] A. Putaja and E. Räsänen, Phys, Rev. B 82, 16533 (2010).

[22] E. Räsänen, T. Blasi, M. F. Borunda, and E. J. Heller, Phys. Rev. B 86, 205308 (2012).

[23] U. Hohenester, Phy. Rev. B 74, 161307(R).

[24] P. M. Poggi, F. C. Lombardo and D. A. Wisniacki, Phys. Rev. A 87, 022315 (2013).

[25] M. G. Bason, M. Viteau, N. Malossi, P. Huillery, E. Arimondo, D. Ciampini, R. Fazio, V. Giovannetti, R. Mannella, and O. Morsch, Nat. Phys. 8, 147 (2012).

[26] G. C. Hegerfeldt, Phys. Rev. Lett. 111, 260501 (2013).

[27] N. Malossi, M. G. Bason, M. Viteau, E. Arimondo, R. Mannella, O. Morsch, and D. Ciampini, Phys. Rev. A 87, 012116 (2013).

[28] A. M. Steane, Phys. Rev. A 68, 042322 (2003).

[29] E. Knill, Phys. Rev. A 71, 042322 (2007).

[30] J. Werschnik and E. K. U. Gross, J. Opt. B.: Quantum Semiclass. Opt. 7, S300 (2005).

[31] J. R. Petta, A. C. Johnson, C. M. Marcus, M. P. Hanson, and A. C. Gossard, Phys. Rev. B 74, 205306 (2006).

[32] L.-X. Zhang, D. V. Melnikov, and J.-P. Leburton, Phys. Rev. B 74, 205306 (2006).

[33] X. Wang, S. Yang, and S. Das Sarma 84, 115301 (2011).

[34] For a while, the trajectory of greater fluence slightly enters into the region with no electron of the CSD. However, this time is short enough to keep the localization of the electron mainly in the DQD and then, it can be can be subsequently recaptured when the trajectory returns to the region of one electron of the CSD.

[35] D. S. Acosta Coden, R. H. Romero, A. Ferrón, and S. S. Gomez, J. Phys. B 46, 065501 (2013).

[36] D. C. Mattis, The Theory of Magnetism Made Simple: An Introduction to Physical Concepts and to Some Useful Mathematical Methods, World Scientific, Singapore (2006).

[37] J. A. Nelder and R. Mead, Computer Journal 7, 308 (1965).

[38] E. Hairer, S. P. Nørsett, and G. Wanner, Solving Ordinary Differential Equations I (2Nd Revised. Ed.): Nonstiff Problems. Springer-Verlag New York, Inc. New York, NY, USA 1993.

[39] Quantum Computation in Solid State Systems, B. Ruggiero, P. Delsing, C. Granata, Y. Pashkin and P. Silvestrini (eds.), ISBN: 0387263322, Springer Verlag 
(Berlin, 2005).

[40] K. D. Petersson, J. R. Petta, H. Lu, and A. C. Gossard. Phys. Rev. Lett. 105, 246804 (2010).
[41] T. Caneva, M. Murphy, T. Calarco, R. Fazio, S. Montangero, V. Giovannetti, and G. E. Santoro, Phys. Rev. Lett. 103, 240501 (2009).

[42] T. Blasi, M. F. Borunda, E. Räsänen, and E. J. Heller, Phys. Rev. B 87, 241303(R). 